\title{
Analyse numérique 3D de la stabilité du front de taille d'un tunnel à faible couverture en milieu frottant
}

\section{R. DEMAGH}

Département de Génje civil

Batra, Algérie rdemaghtyahoo.fr

\section{F. EMERIAULT}

LGCIE

INSA-Iyon

Bat JCA Coulomb,

34 avenue des arts

69621 Villewhanne Cedex

Fabrice.Emeriaut@insa-lyon.fi

\section{S. BENMEBAREK \\ LRGCB-Département de Génie civi Biskra, Aigérie}

Les méthodes de calcul à la rupture proposent des solutions au problème de soutènement du front de taille d'un tunnel, soit à l'aide d'une approche stalique (par l'întérjeur', soit avec une approche cirématique (раг l'extérieur). Ces méthodes, appropriées aux matériaux standard, imposent les mécanismes de rupture en surestimant la ditatance des sols frottants. Dans cet artícle, on s'intêresse au calcul en déplacements, à l'aicle du code FLAC en différences finjes explicites, de la stabilité cu front thun tunnel à faible couverture creusé dans un sol frottant. L'effet du caractère non associé de la règle d'écoulement sur les pressions linites et les mécanismes de rupture est examiné. Deux modes de rupture sont anałysés, correspondant aux cas cle la poussée (collapse) et de la butée (blow-out). Les résultats du calcul en déplacement sont également utilisés pour évaluer la pertinence de modèles analytiques basés sur l'approche cinématique de l'analyse limite.

Mots-clés : front de taille, sols frottants, analyse limite, mécanisme tridimensionnel, méthode numérique.

\section{D Numerical analysis of face stability of shallow tunnel in cohesionless material}

The vield design theory proposes some solutions to the problem of tunnel face stability either by a static approach (lower bound! or kinematical approach I upper bound). The failure mechanisms considered in these methods, based on an associated flow rule. ane imposed. In this paper, the face stability of shallow tunnel in cohesionless material is investigated by 3D numerical simulation using FLAC3t code. The effect of a non-associated flow rule on the critical pressures and on the failure mechanisms are examined. The relevance of analytical models based on the kinematical approarh of the limit analysis is evaluated also.

Koy words : tumel face, frictional soil, imit analysis, threedimensional mechanism, numerical method. 


\section{Introduction}

En zone urbaine, concevoir un tunnel (creusé au bouclier à front pressurisé dans les terrains meubles) impose une maîtrise totale de la stabilitê du front de taille, particulièrement à faible profondeur. Se basant sur des mécanismes bidimensionnels, le problène de la stabilité du front a été étudié pour la première fois par Atkinson et Potts (1977) en terrain purernent frottant, ensuite par Davis et al. (1980) en terrain purement cohérent. En milieu frottant-cohérent, on connait essentiellement en bidimensionnel les résultats obtenus par Muelhaus (1985), Leca et Panet (1988), Chambon et Corté (1990), en tridimensionnel, Leca et Dormieux (1990), Soubra [2000), Wong et Subrin (2006). Tous se réferent au calcul à la rupture par analyse limite et proposent des solutions analytiques (ou sous forme d'abaques). Le calcul de la charge de rupture se fatt avec deux approches complémentaires ; une approche statique quí donne la bome inferieura de la pression linite et une approche cinématique qui fournit la bone superieure.

Lapproche cinématique est fondée sur la règle de normalité de Hill qui reste sévère pour les matériaux non standard $(\psi<\varphi$ ). La conséquence de lapplication de cette règle à un sol frottant, avec un angle de frottement interne $(p$, est que son écoulement plastique sera accompagné par une expansion de volume caractérisée par un angle de dilatance $\psi=$. Cependant, les sols frottarts s'averent expérimentalement se dilatel moins que ce qui est prévu par la règle de normalité et doivent donc être considérés comme non standard. Un sable lâche constitue, à ce titre, un cas extrême (maté riau contractant pour lequel $\psi=0$ ). La non prise en compte de la dilatance des matériaux non standards est donc la principale limite de la méthode.

Par ailleurs, la bone supérieure dépend aussi du mécanisme de rupture imaginé, ce qui constitue une autre limite. Des techniques de régularisation de la néthode cinématique ont été proposées permettant de pallier à cette dernière difficulté (Pastor et al., 2000 ; Lyamin et Sloan 2002 ; module LIMI de CESAR ; Droniuc et al., 2005a, 2005b).

Cependant, les résultats ne permettent pas toujouj's d'aboutir à une quantification précise du risque d'instabilité du fait de l'écart important qui subsiste entre les approches statique et cinemalique.

Le recours aux méthodes de calcul en déplacement. qui s'affranchissent des hypothèses des méthodes analytiques, est aussi ur moyen d'évaluer leur pertinence. Le calcul en déplacement permet de représenter des complexités diverses cui peuvent être liées soit à des configuratinns génmétriques (différentes couches de sol en particulier), soit à des chargements particuliers (forces d'écoulement par exemple). Il permet aussi de prendre en compte plus finement le comportement des sols (caractère non associé, comportement pré-rupture, anjsotropie...) et de nimposer aucune contrainte sur la forme et l'ampleur du mécanjsme de rupture. Cependant, outre le caractere prohibitif des temps de calcul liés à l'analyse d'une configuration particuliere ou de la réalisation d'une etude paramétrique complète, il faut mentionner l'attention toute particulière qu“il faut apporter à la définition des conditions aux limites imposées et à leur description numérique (précision des pas de calculs, critères ce convergence...).

Dans cế article, on šintéresse à la stabilité tricimensionnelle du front de taille en milieu frottant, avec un matériau standard ( $\psi=q$ ) et non standard (1e cas extrême $\psi=0$ a été retenu dans cette étude). Ceite analyse est menée dans le cas de la poussée et de la butée des terres, communement appelés collapse et blowout. Les résultats du calcul er déplacements obter.us à l'aide du code FLAC en différences fines sont conparés aux principales approches cinematiques tridi= mensionnelles proposées dans la littérature et qui sont rappelées dans le paragraphe?.

\section{2}

\section{Approche par analyse limite}

\section{1}

\section{Cas des matériaux standard $(\psi=\varphi)$}

Le problème est idéalișé avec un tunnel de diarnètre D creusé dans un milieu homogéne et pesant de poids volumique $\gamma$, sous une couverture C. Le matériau est standard ( $\psi=\varphi$ ), ses paramètres de résistance sont la cohésion c et l'angle de frottement interne $\varphi$. Une pression uniforme os simule une surcharge éverituelie en surface.

Dans le cas de la poussée, Leca et Domieux (1990) ont imaginé un mécanisme de rupture tridimensjonnel, composé de deux blocs tronconiques ă un degré de liberté $\alpha$, le cône supérieur étant maintenu vertical. L'optimisation faite sur a conduit à une borne supérieure pour $\alpha^{*}=49-p / 2$ (en degrés), figure 1a. Soubra (2000) a étendu ce mécanisme en intercalant entre les deux blocs ume zone de cisaillement radial. appelée log sandwich et a libéré l'axe vertical du bloc supérieur (Fig. 1b). La zone log sandwich est approchée avec (n 2) blocs tronconiques. Soubra (2000) montre que pour $n \geq 5$, la pression limite reste la mème. Leca et Dor-

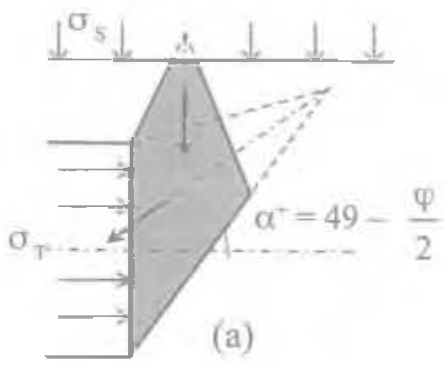

(a)

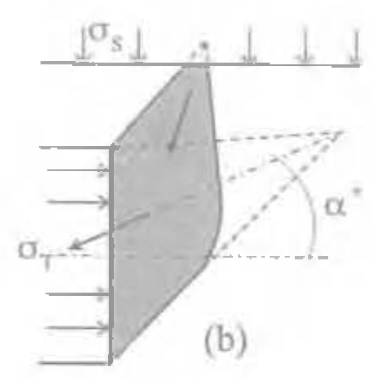

(b)

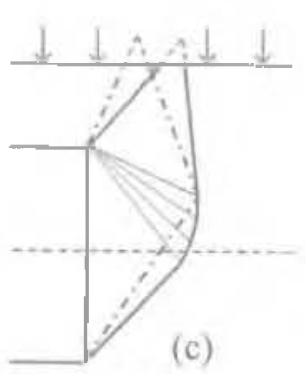

(c)

FIG 1. Mécanismes $\varphi=20$ degrés et $C / D=0,4$ : (a) Leca et Dormieux (1990) ; (b) Soubra (2000) ; (c) superposition. Collapse mechanism $\varphi=20$ degrees and $\mathrm{C} / \mathrm{D}=0.4$ : (a) Leca et Dornieux (1990); (b) Soubra (2000) ; [c] superposition 
mieux 11900$)$ comme Souhra $20 n n$ snnt parvenus à la même formulation de la borne supérieure, donnée par les équations (1) et (2). Les coefficients $N_{s}{ }_{s}{ }^{*}, N_{c}{ }^{c *}$ et $N_{c}{ }_{c}{ }^{*}$ ne sont en revanche pas les mêmes pour les auteurs:

avec

$$
\sigma_{T} \geq \sigma_{S} N_{S}^{C+}+\gamma D N_{F}^{C+}+c N_{C}^{c+}
$$

$$
N_{c}^{C+}=\frac{N_{s}^{c *}-1}{\operatorname{tg} \varphi}
$$

Chambon et Corté (1990) ont proposẻ un mécanisme de rupture bicimensionnel avec un monobloc rigide, délimité par deux arcs de spirales logarithmigues (Fig. 2a). Plus tard, Wong et Subrin (2006) l'ont adapte au cas tridimensionmel et ont proposé un mécanisme original en forme de corne de rhinocéros, construit à partir d'arcs de spirales logarithmiques (Fig. 2b).

Dans le cas de la butée, Leca et Dormieux (1990) ont considéré un mécamisme monobloc, l’optimisa tion faite sur $\alpha$ donne une borne supermeure pour $\alpha=$ 49 degrés (Fig. 3a). Soubra (2000) a proposé uл mécanisme inverse à celui de la poussée (Fig. 3b). Leca et Dormieux (1990) comme Soubra (2000) ont tournj la même formulation de la borne supêtreure donnée par les équations (3) et (4) avec des coefficients $\mathrm{N}^{*}, \mathrm{NB}^{*}$ et Noropres à chaque mécanisme

avec

$$
\sigma_{T} \leq \sigma_{S} N_{S}^{\beta+}+\gamma D N_{Y}^{8+}+c N_{C}^{8 *}
$$

$$
N_{C}^{H+}=\frac{N_{S}^{B+}-1}{\operatorname{tg} \Phi}
$$

Dans cet article, les résultats du calcul en déplacements à l'aide de ELAC ${ }^{30}$ sont comparés aux résu' tats du calcul par analyse limite foumis par Leca et Dormieux (1990) et Soubra (2000). Ceux-ci présentent

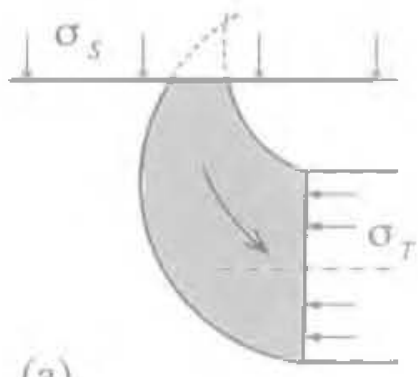

(a)

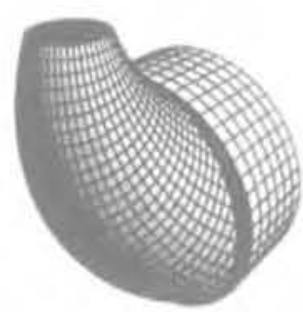

(b)

FG. 2 Mécanisme débouchant: (a) Chambon et Corté (1990); (b) Wong el Subrin (2006). Collapse mechanism: iaj Chambon \& Corté (1990); (b) Wong et Subrin (200b). l'intêrêt d'avoir été développés avec une homogếnéité d'approche en poussête et en butée, même si l'anàlyse bibliographique montre que le mécanisme, en poussée uniquement, de Wong et Subrin (2006) affine la borne supérieure.

\section{2}

\section{Cas des matériaux non standards $(\psi<\varphi)$}

Le problème de la stabilité du front de taille dans Ies matériaux non standard n'a pas encore été étudié. En s'intéressant au problème de la capacité portante des fondations filantes, Drescher et Detournay (1993) ont montré que la charge limite pour แл matériau non standard correspond en fait à une résistance réduite liée à la non-associativité de la règle d'écoulement. Cette approche donne une bonne approximation de la capacité portante des fondations supenficlelles pour les cas anailysés, $\psi=0$ et $\psi=\varphi / 2$. La charge limite fournie par un calcul en déplacement pour un matériau non associé est comparée à celle évaluée avec les équations (5) a (7). La différence est de $12 \%$ lcas $\varphi=20$ degrés avec $\psi=0$ ) et de $20 \%$ (cas $\varphi=40$ degrés avec $\psi=$ 20 degrés). Les paramètres réduits pour un matériau de Coulomb sont :

avec

$$
\begin{aligned}
& \operatorname{tg} \varphi^{\circ}=\eta \operatorname{tg} \varphi \\
& c^{*}=\eta C \\
& \eta=\frac{\cos \psi \cos \varphi}{1-\sin \psi \sin \varphi}
\end{aligned}
$$

\section{3}

\section{Cas d'étude et exploitation}

\section{1}

\section{Configuration étudiée}

On considère un tunnel de diametre $\mathrm{D}=6,5 \mathrm{~m}$, creusé sous une couverture $C$ variable. On s"intéresse à Lin matériau purement frottant, le massit est homogène et la cohésion est nulle. Le poids volumique y vaut $16 \mathrm{kN} / \mathrm{m}^{3}$ (en reférence aux essais en centrifugeuse de Chambon et Corté, 1990), la surface du massif est soumise à une surcharge $\sigma_{\mathrm{S}}=25 \mathrm{kPa}$. Le modéle géomé-
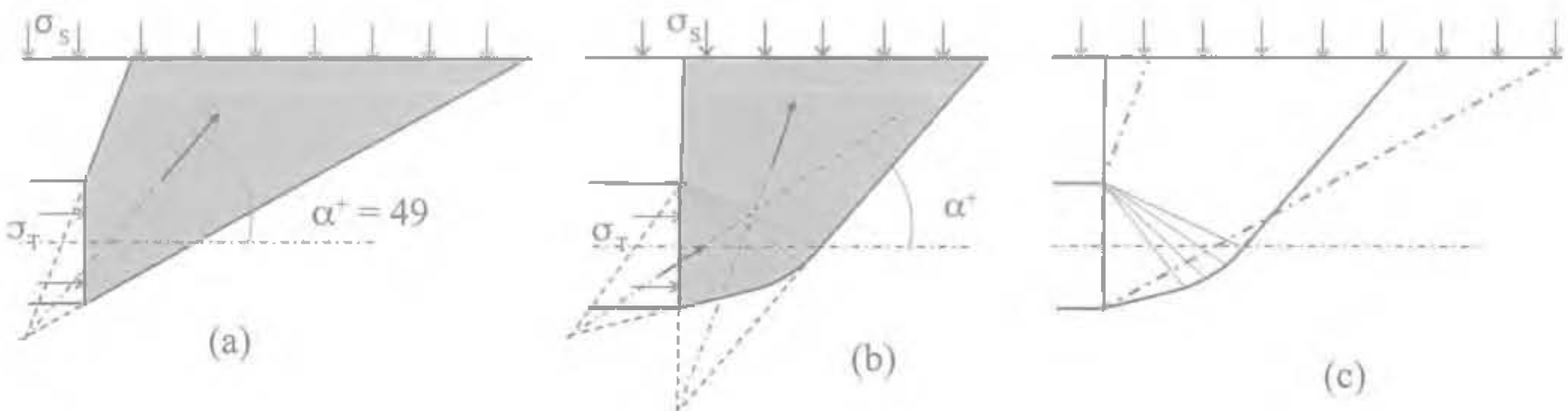

(c)

F5. 3

Mécanismes $q=20$ degrés et $C / D=1$ : (a) Leca et Dormieux (1990) ; (b) Soubra (2000) ; (c) superposition. Coliapse mechanism $\varphi=20$ degrees and $\mathrm{CD}=$ ] : [a] Leca \& Dormleux [1990); (b)] Soubra [2000): (c) superposition. 
trique a pour dimensions $60 \mathrm{~m} \times 60 \mathrm{~m} \times 30 \mathrm{~m}$ (Fig. 1b). Le mailiage ast composé d'environ 25000 ncuds, il est généré avec lés éléments volumiques de FLAC ${ }^{30}$ (brique â huit noeuds). Les conditions aux limites classiques sont appliquées : déplacement horizontal nul sur les faces verticales et déplacement vertical nul tै fa base. Le sol est modélisé en élasto-plasticité avec le critère de rupture de Mohr-Coulomb. Un état de contraintes géostatiques est initialisé avec un coefficient des terres au repos $K_{0}=0,5$. Le module élastique est $E=150 \mathrm{MPa}$ et le coefficient de Poisson $v=0,3$. La surcharge $\sigma_{\text {s }}$ est appliquée graduellement par incrément de $2,5 \mathrm{kPa}$. La stabilité du front est assurée par une pression uniforme $\sigma_{\mathrm{T}}$, initialement égale à la contrainte horizontale naturelle. Pour simuler un revêtement parfaitement rigide, les nceuds situés sur la frontière de l'excavation sont fixés.

\section{2}

\section{Modèles de calcul}

Les simulations ont été effectuées pour chacun des modes en poussée et en butée. Elles correspondent aux cas : $C / D=1$ avec $\varphi$ variable $20 \leq \varphi \leq 40$ (en degrés) et $\varphi=30$ degrés avec un rapport C/D variable $0,5 \leq \mathrm{C} / \mathrm{D}$ $\leq 2$. Pour rendre compte du phénomène de dilatance des matériaux frottants. les deux cas suivants sont pris en considération : matériau standard $(\psi=\emptyset)$ pour permettre une comparaison avec les pressions limites données dans la littérature et matériau non standard ( $\psi$ = 0) pour examiner l influence de la dilatance dans un cas extreme.

\section{3}

\section{Paramètres de calcul et critères de convergence}

Une étude de sensibilité de la pression limite vis. à-vis des pamamétres de calcul a été faite dans le cas de la poussée, sur̃ un matériau standard avec $\mathrm{C} / \mathrm{D}=1$ et $\varphi=30$ degrés. Les deux paranetres concemés par cette étude sont le nombre de pas de calcul de chaque palier de presşion appliquée au front 15000 pas en référence aux recommandations de la notice de FLAC ${ }^{30}$ ou
10000 ct la weur de lincrénent de dininution de la pression, 2,5 ou $1 \mathrm{kPa}$.

Pour tous les cas testés (Fig. 5a), le dernier palien de pression pour lequel on observe encore la stabilité du front est le même (12,5 kPa en l'occurrence). A partit de ce palier", une réduction de la pression de $0,5 \mathrm{kPa}$ provoque une rupture (ce qui correspond à une précision maximale de $4 \%$ sur la pression limite). D’une façon globale, la précision des résultáts du calcul en déplacenent, dans le cas de la rupture par poussée. est au maximum comprise entre 2 et $6 \%$, la recherche d'une meilleure précision, jugée peu utile, entraînerait en revanche des temps de calcul importants.

En outre, les différents chargenents testés ont conduit à une réponse qui est pratiquement la même en termes de déplacement final du front. La figure ba montre l'extrusion axiale du front (rapportée au diamètre D de l'excavation) enregistrée a la fin du calcul de chaque palier en fonction de la pression appliquée au frorit. Cette figure montre, en particulier, cue la rupture du front est effective après un déplacement relatif de lordre de $1 \%$.

La butée quand à elle est simulée par une incrémentation de $25 \mathrm{kPa}$ avec 5000 pas de calcul associés à chaque palier de pression appliquée au front (recommandiations de FLAC ${ }^{3 D}$ ). La précision sur les pressions limites est au maximum comprise entre 0,5 et $3 \%$, ce qui justifie par ailleurs ce choix.

Cette façon de procéder, même si elle entraîne des temps de calculs importants, est préférable du point de vue physique an une simulation basée sur บл contrôle en déplacement.

Typiquenent, avec un processeur Intel Pentium 4 et 3 Go de vitesse d'horloge, les temps de calculs jusqu'à la rupture, pour le cas d'un matériau standard avec C/D $=1$ et $\varphi=30$ degrês, sont de 15 heures pour le mode poussée et de 45 heures pour le mode butée, le malllage étant composê d'environ 25000 nouds. Les temps de calculs augmentent rapidement avec l'angle de frottement interne (et donc avec la dilatance) : a titre d'exemple, en mode butée pour le cas C/D = 1 et $\varphi=40$ degrés, il faut 200 paliers de $25 \mathrm{kPa}$ pour atteindre les $5 \mathrm{MPa}$ nécessaires à la rupture, za raison de 5000 pas de calcul par palier, it faut au moins 4 jours de calcul.

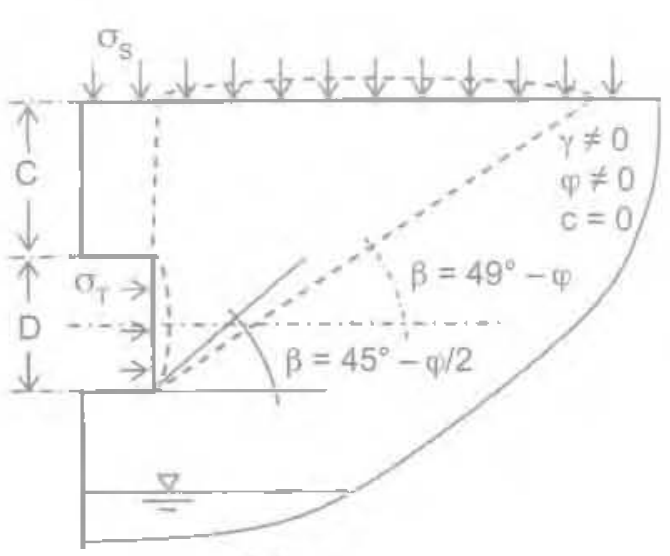

(a)

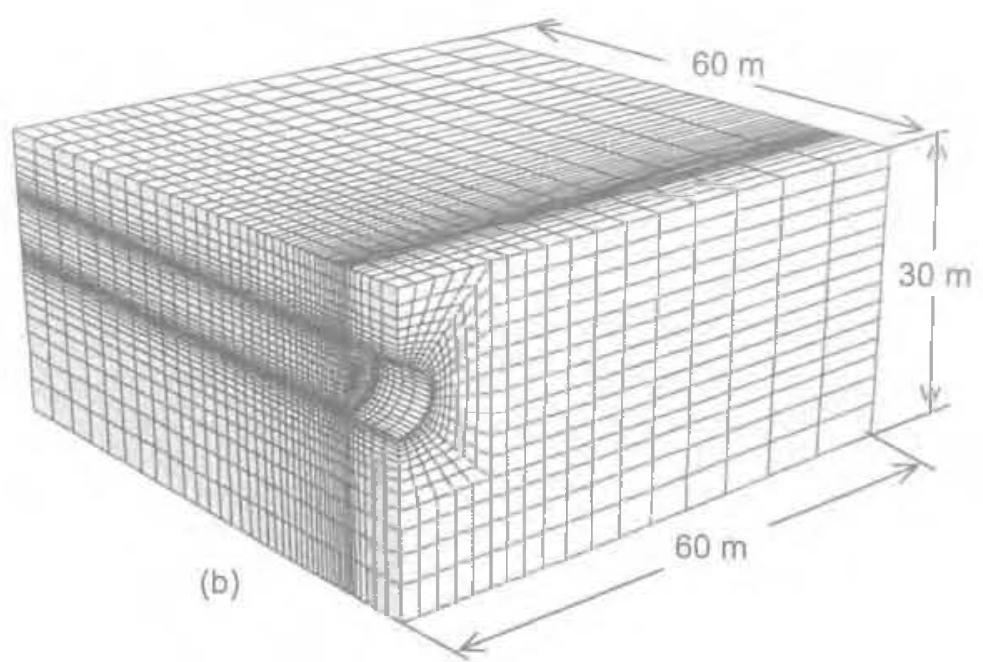

(b)

FIG. 4 Cas étudié : (a) en butée d'après Leca et Dormieux (1990) ; (b) maitlage 3D utilisé. Study case : (a) blow-out mode Leca 8 Dormieux (1990), (b) 3D mesl used. 


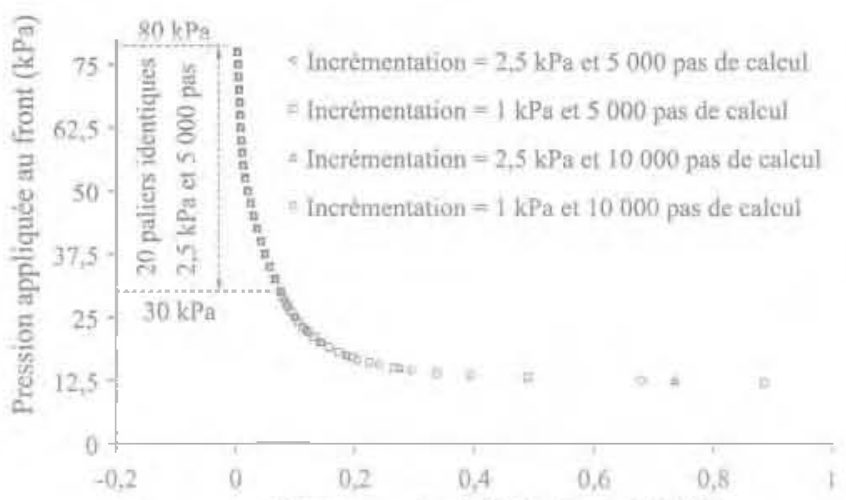

(a)

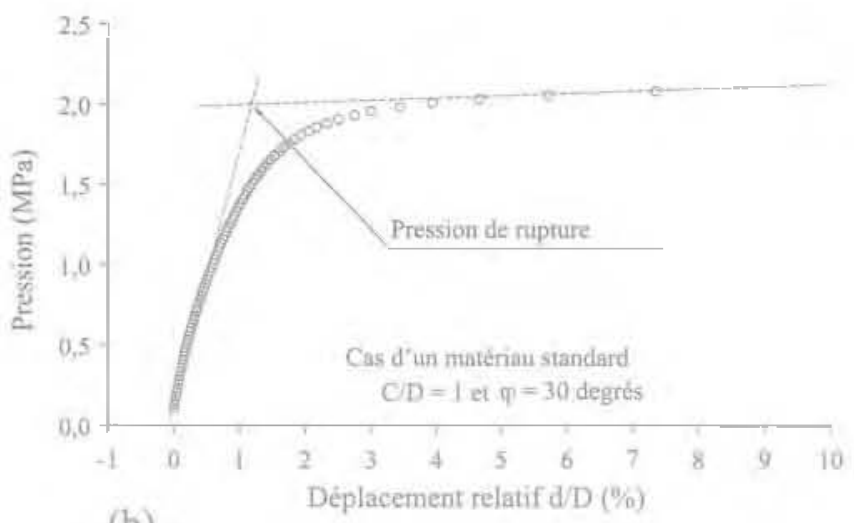

(b)

FIG. 5 (a) Étude de sensibilité ; (b) critère en déplacement. Criterle of fallure: (al sensitivity study : lb) displacement criteria.

\section{4}

\section{Critères de rupture du front de taille}

Pour apprécier la pression de rupture. Denagh et al. (2005) et (2006), dans des travaux précédents, ont proposé une méthode basée sur un calcul en déplacement à l'aide de FLAC ${ }^{35}$. La courbe de déplacement du front en fonction de la pression $\sigma_{\mathrm{T}}$ a été exploitée comme indiqué sur la figure $5 \mathrm{~b}$. Cette approche relativentent rapide permettait un calcul paramétrique mais avait tendance à donner une valeur trop conservative.

Pour cette analyse, deux critêres propres au code FLAC BD $^{3 D}$ sont utilisés pour juger de l'apparition de la rupture. Le premier critère est observé lorsque la force maximale non équilibrée (maximum unbalanced force) converge vers une valeur non nulle (égale à quelques clizaines de Newton, figure $6 \mathrm{~b}$ ). Le second critère concerne la vitesse axiale d'un nceud appartenant au front (le centre du tunnel par exemple). Pour le palier équilibré précédent la rupture, le champ de vitesse est diffus à travers tout le massif et la vitesse est quasi nulle (valeur comprise entre $10^{-11}$ et $10^{-12} \mathrm{~m} / \mathrm{pas}$ de calcul). Au pálier suivant (correspondant à la mupturel, cette vitesse enregistre un saut et ne peut plus converger vers une valeur constante (Fig. Gb]. Ce saut de vitesse est accompagné par l'apparition brutale d'un champ de vitesse locallsé (Fig. Ga). On estime que la rupture est atteinte a partir d'une vitesse de $10^{-5} \mathrm{~m}$ pas de calcul, ce quil correspond à un déplacement axia! de $10 \mathrm{~cm}$ tous les 10000 cycles de calcul.

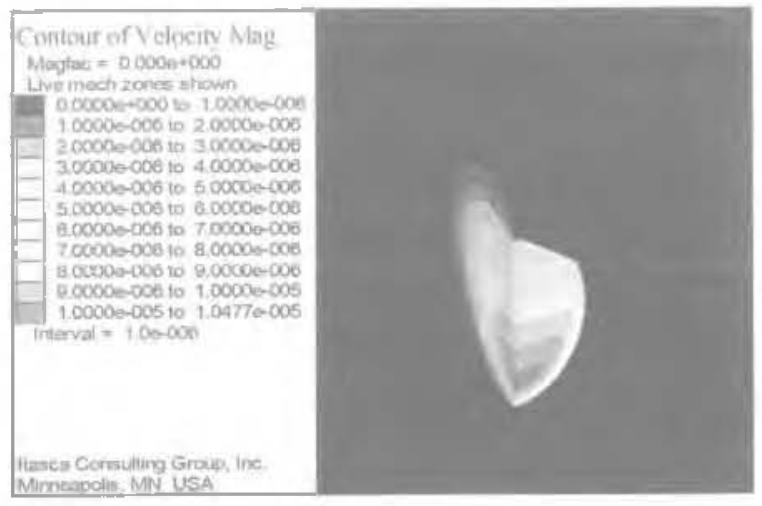

(a)
L'application simultanée de ces deux critères permet une évaluation précise de la charge limite correspandant à la pression de rupture.

\section{Résultats et interprétation}

Les résultats des simulations sont donnés sous forme de courbes représentant la pression limite en fonction du rapport C/D et de l'angle de frottement $\varphi$. Les équations (1) a (4) permettent le calcuJ de la borne supérieure des pressions limites évaluées par Leca et Dormieux (1990) ainsi que par Soubra \{2000).

Dans le cas de la poussée, le mécanisme à cinq cônes de Soubra (2000) apporte une amélioration de 6 à $9 \%$ par rapport au mécanisme à deux connes de Leca et Dormieux (1990). La différence entre les pressions limites obtenues avec un calcul en déplacement et celles évaluées à partir du mécanisme à cinc cônes est infếrieure à $10 \%$. On note mème une bonne concordance pour le cas d'un matériau standard avec $\varphi=30$ degrés (Fig. 7a). Les pressions limites données par le mécanisme à cing cônes constituent bien une borne supé. rieure quels que soient le rapport CD [Fig. 7a) et l'angle de frottement $\varphi$ (Fig. 7b). Par ailleurs, les pressions limites calculées avec FLAC ${ }^{35}$ sont indépendantes du rapport C/D (Fig. 7a), ce qui est en accord avec l'équation (1) pour laquelie les paramètres $\mathrm{N}_{y}^{c *}$ et $\mathrm{N}_{\mathrm{c}}^{\mathrm{E}}$ fournis

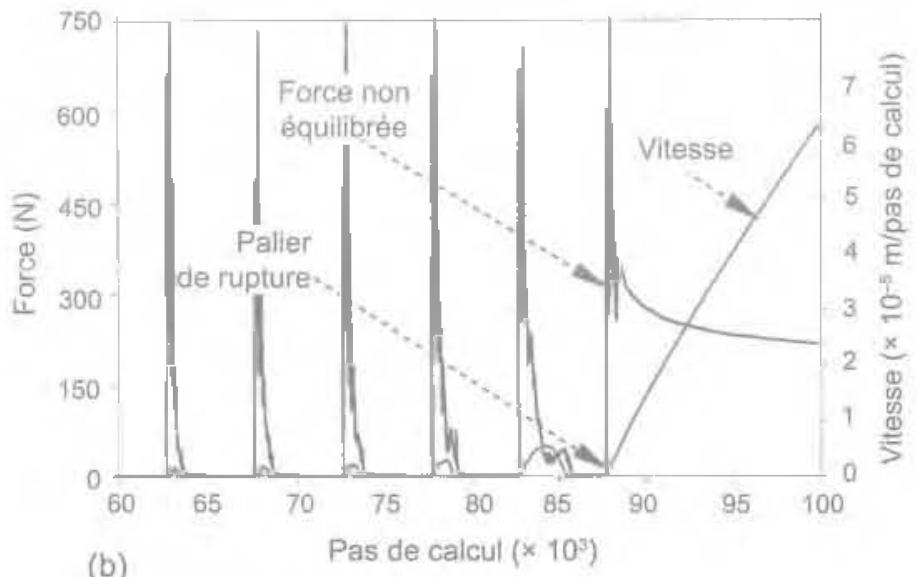

(b)

Critères de rupture du front : (a) contour du champ de vitesse ; (b) historiques de la force maximale non équilibrée et de la vitesse.

Criteria of failure : (a) contour of velocity i (b) histories of maximum unbalanced fonce and velocity. 
Паг l'analvse limite snnt respertivement constant et nut pour le cas $C / D \geq 0,5$ et $\varphi \geq 20$ degrés.

Pour un matêriau non standard (ici purement contractant $\psi=0$ ), les pressions limites obtenues aved FLAC ${ }^{30}$ restent supérjeures de 4 à $7 \%$ à celles calculées avec un matériau standard (Fig. 7a et 7b).

Ces mêmes résultats ont été comparés avec l'ap. proche par analyse limite non associée proposée par Drescher e: Detournay (1993). On note une grande différence entre les deux approches (jusqu $3,2 \mathrm{kPa}$ au maximum, soit $30 \%$ ). De plus, pour un angle $\varphi \geq$ 23 degrés anviron, les pressions limites données par l'analyse limite non àssociée sont supérieures aux pressions limites obtenues avec un calcul en dêplacement et de ce fait ne peuvent constituer une borne supérieure pour le cas extrêne $\psi$ ' = 0 (Fig. 9â). Ainsi, pour l'évaluation de la pression limite dans le cas d'un matériau non standard, on préférera l'approche originale de Soubra (2000) avec $\psi=\emptyset$

Dans le cas de la butée, le mécanisme à cing cônes de Soubra (2000) améliore jusqu'à $50 \%$ les pressions limites donnees par le mécanisme monobloc de Leca et

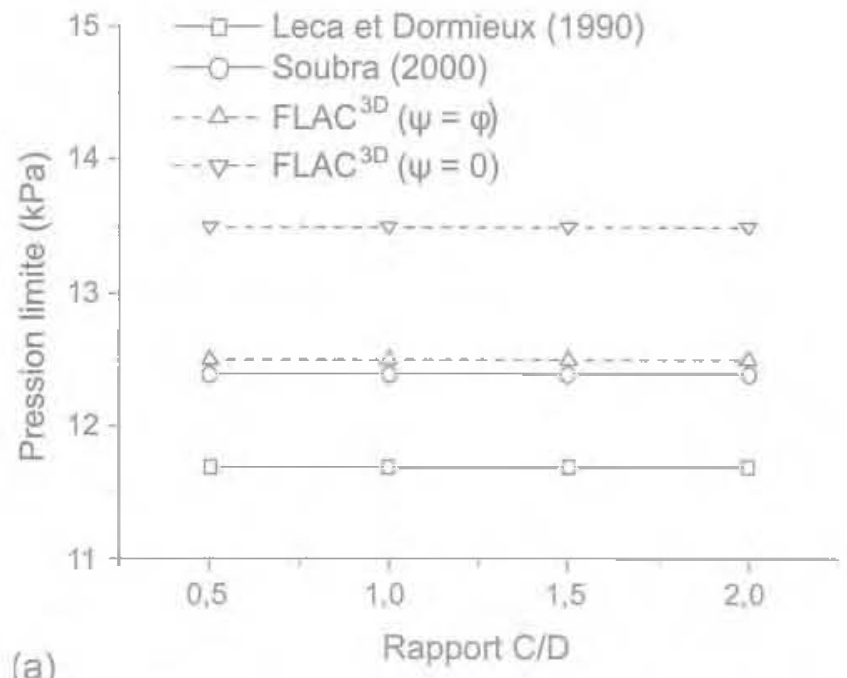

Dommeux [19and (Fing 8a et Bb) Pour un matériau standard, les pressions calculées à partir du mécanisme de Soubra (2000) sont supérjeures de 13 à $40 \%$ aux pressions limites fournies par un calcul en déplacement.

Pour un matériau non standard, les calculs FLAC ${ }^{35}$ fournissent des valeurs de pression limite inférieures de 10 à $40 \%$ à celles calculées avec un matériau standard. Cette différence, contrairement au cas de la poussée (4 à $7 \%$ ), montre l'influence de la dilatance sur la butée.

La figure gb montre que, pour un matériau non standard, l'approche par analyse limite non associée proposée par Drescher et Detournay (1993) fournit des pressions limites supérieures de 10 à $20 \%$ à celles obtenues avec un calcul en déplacement el qu'elle peut donc constituer une bonne approximation de la borne supérieure pour les matéríux non standard, du moins pour le cas extrême $\psi=0$.

La validation de l'approche de Drescher et Detournay (1993) avaił été effectuée à partir de calcul de capacité portante cle semelles de fondation filantes. U est intéressant de noter que cette approche semble être pertinente

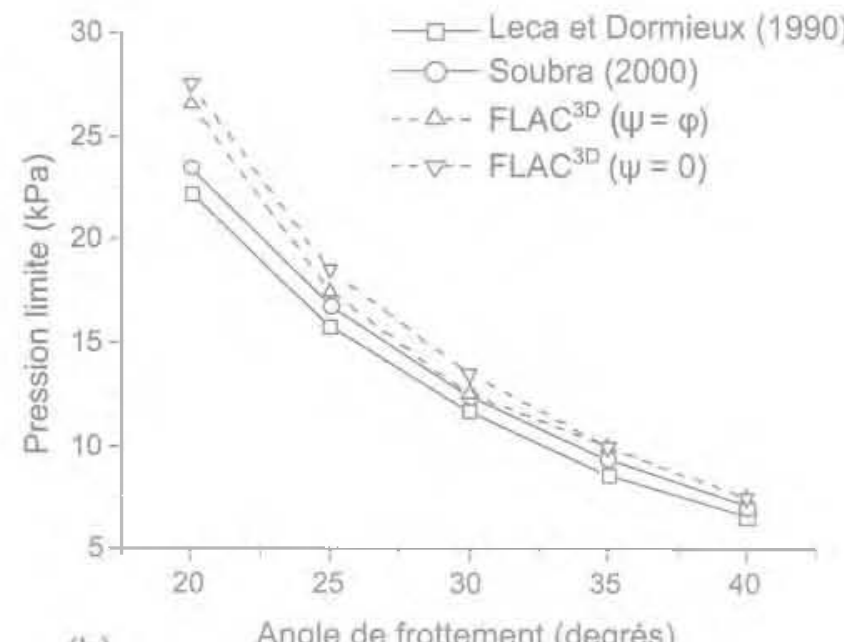

(b)

Angle de frottement (degrés)

*G. 7 Borne 5Lpérieure en poussée : (a) $\varphi=30$ degrés ; (b) $\mathrm{C} / \mathrm{D}=1$. Upper bound for collapse : (a) $\varphi=30$ degrees : $(\mathrm{b}) \mathrm{C} / \mathrm{D}=1$.
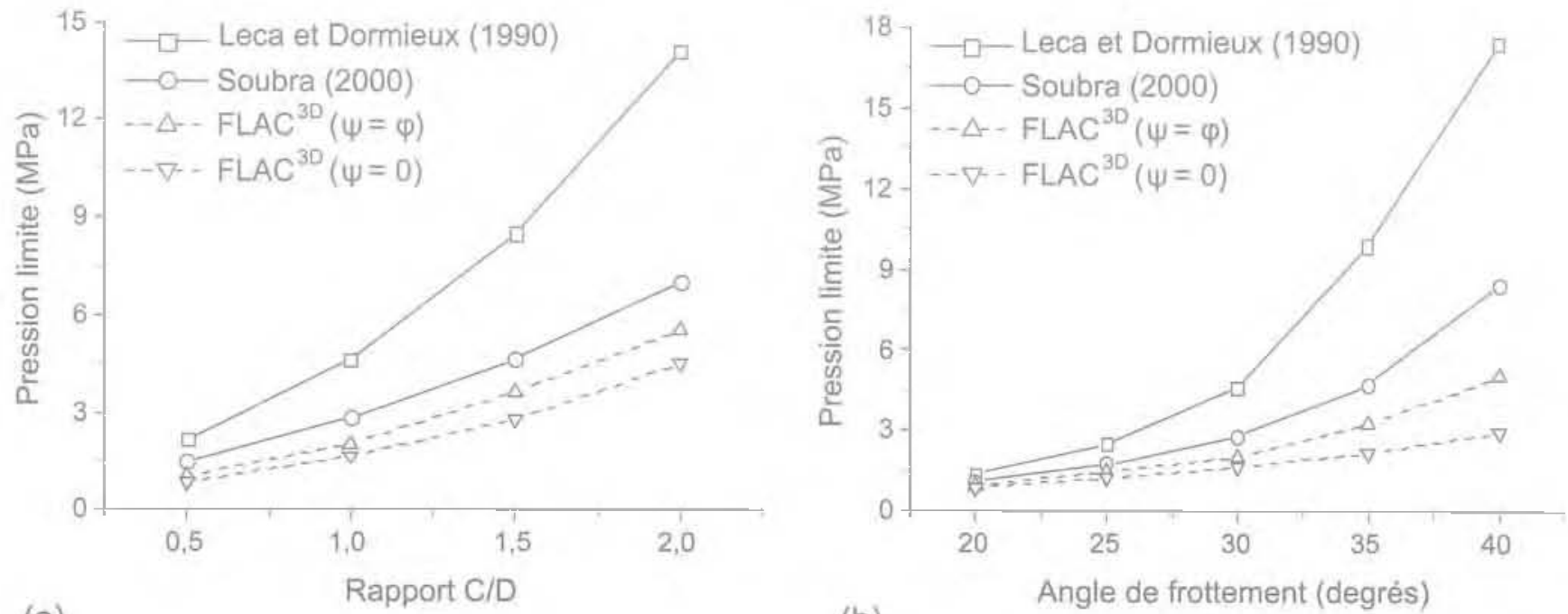

(a)

FIG. \& Borne supérieure en butée : (a) $Q=30$ degrés ; (b) $C / D=1$. Upper bound for blow-out : (a) $p=30$ degrees; $(\mathrm{b}) \mathrm{C} / \mathrm{D}=1$. 


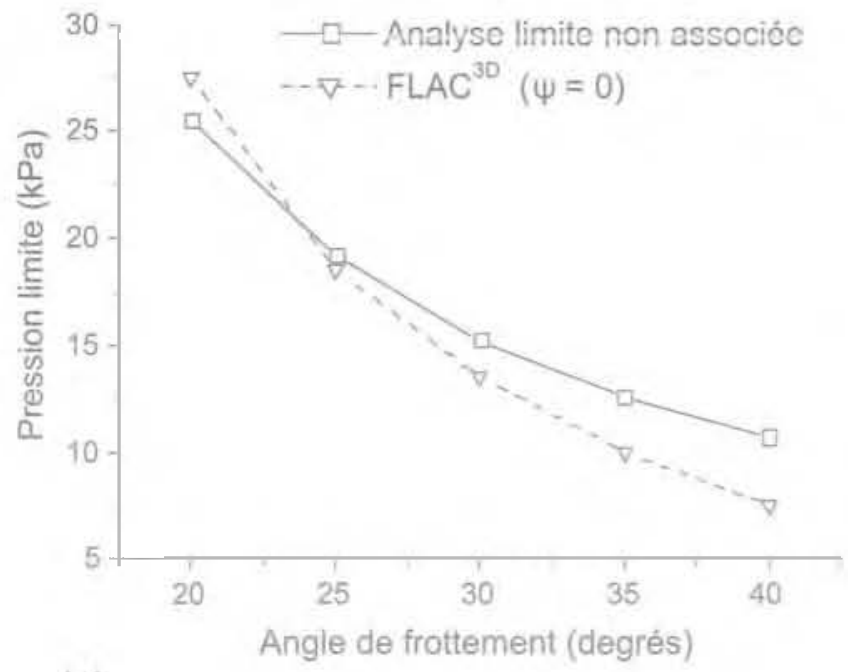

(a)

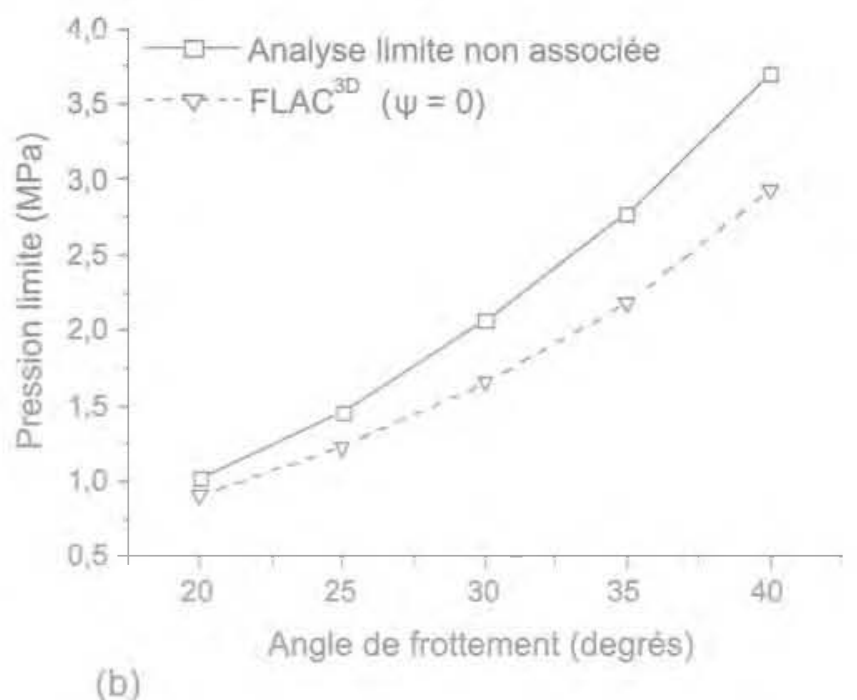

(b)

FG. 9 Comparaison de ta pression limite donnée par FLAC ${ }^{3 D}(\psi=0)$ et l'analyse limite non associée donnée par l'équation (5) pour $(\psi=0$ ) : (a) en poussée; (

Comparison of Iimit pressure from FLAC.30 and equation (5) : (a) collapse ; (bl blow-out.

dans le cas d"une sollicitation simtlaíre, celle de la rupture en butée du front d'un tunnel alors qu'elle donne des résultats non satisfaisants pour une rupture en poussée.

\section{5}

\section{Analyse des mécanismes de rupture}

Les résultats de la section 4 sont ici analysés sous l'angle des mécanismes de rupture. Pour cela, on a superposé le champ de vitesses obtenu par le calcul en déplacement et celui construit à partir du mécanisme à cing blocs de Soubra (2000).

Dans le cas de la poussée, pour un matériau stardard, les figures 10 a et 10 présentent les mécanismes obtenus pour $\mathrm{C} / \mathrm{D}=1$ et pour deux valeurs de $\varphi$. Le mécanisme obtenu pour $\mathrm{n}=10$ degrés débouche en surface alors que pour $\varphi=25$ degrés celui-ci est non débouchant. Ces exemples confirment les résultats de l'approche par analyse limite cui prédisent pour tout le domaine définj par $\mathrm{C} / \mathrm{D} \geq 0.5$ et $\varphi \geq 20$ degrés, des mécarismes de rupture en poussée nor débouchant. Dans les deux cas de figure, le champ de vitesse obtent avec $\mathrm{FLAC}^{3 \mathrm{D}}$ est bien encadré par le mécanisme à cinq blocs, avec cependant une meilleure approximation pour le cas d'un mécanisme débouchant. Le mécanisme lié aux forces de surface (fournissant le coefficient $\mathrm{N}_{\mathrm{s}}$ jest par ailleurs légèrement plus performant que celui qui est définj à partir des forces de gravité (donnant le coefficient $N_{V}$ ).

Lorsque le matériau est non standard (figure 10c avec $C / D=1, \varphi=30$ cegrés et $\psi=0$ ), le calcul en déplacement fournit un mécanisme de rupture débouchant et qui présente une forme cylindrique dans sa partie supérieure. L’approche par analyse limnite modifiée suiwant les propositions de Drescher et Detournay (1993) donne en revanche un mécanisme non débouchant qui, du fait de la valeur non nulle de $\varphi^{*}$, est de forme conique à proximité de la surface. Il apparait donc intéressant de proposer une autre approche par analyse limite non associée pour la détermination des caractéristiques réduites $c^{*}$ et $\varphi^{*}$ qui permette, en particulier Lorsque $\psi=0$, d'avoir $\varphi^{*}=0$ et $c^{*}$ non nutle, conditions nécessaires pour obterii un mécanisme de rupture par analyse limite cylindrigue dans sa partie supérieure et donc débouchant en surface.
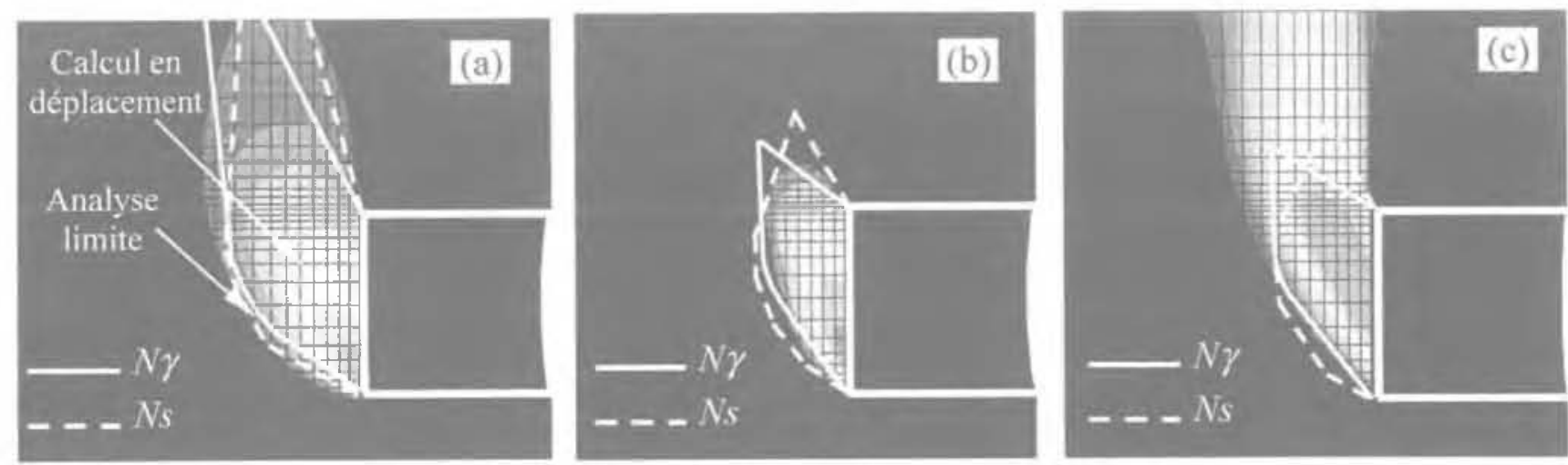

FG 10 Mécanismes en poussée $C / D=1$ : (a) débouchant $\psi=\varphi=10$ degrés ; (b) non débouchant $u=\varphi=25$ degrés : (c) matéeriau non standard $\psi=0$ et $\phi=30$ degrés $(0 *=26,6$ degrés).

Collapse failure mechanism CD $=1:$ la) $\psi=\varphi=10$ degres; $(b) \psi=\varphi=25$ degrees; (c) nor-standard material $\psi=0$ and $\varphi=30$ degrees [ $\varphi^{*}=26.6$ degrees; 
De plus, il est intéressant da noter que, pour le cas des matériaux standard $(\psi=\varphi)$, la rupture obtenue par le calicul en déplacement semble plus présente en radier du tunnel (Fig. 6a, 10 a et $10 b$ ), ce cui est logique pour un matériau pesant. Dans le cas des matériaux non standard " $U$ = 0), on observe wne vitesse maximum au niveau de l'axe du tunnel. Le mécanisme de rupture translationmel retenu par l'analyse limite ne permet pas de rendre compte de ce phénomène.

Dans le cas de la butée pour un matériau standard, le mécanisme à cing blocs encadre très largement le champ de vitesses capté juste après la rupture (Fig. 11a), ce qui explique en partie la marge de 13 k̀ $40 \%$ entre la borne supérieure donnée par le mécanisme à cinq cones et les pressions limites fotrnies avec uñ calcul en déplacement (Fig. 8a et 8b). Globalement, le mécanisme de Soubra prédit un bloc en rupture de volume plus important gue celui obtenu avec FLAC ${ }^{30}$. Les mécanismes de rupture associés aux termes de surface et de gravité iresponsables des coefficients $N_{\text {g }}$ et $N_{j}$ ) sont globalement identiques.

Le constat est le même pous un matériau non standard (Fig. 11b). Lanalyse limite modifiêe suivant les propositions de Drescher et Detournay (1993) conduit a un mécanisme de rupture encore plus important que celui fourni par un calcul en déplacement, de forme cylincrique dans sa partie supérietre, ce qui, comme dans le cas de la rupture par poussée, incite à proposer une nouvelle approche de l'analyse limite non associé pour le calcul des caractéristiques réduites tp et $c^{*}$.

Dans les matériaux frottants (avec ou sans cohé sion), la méthode de la borne supérieure de l'analyse limite impose un mécanisme translationnel de cônes rigides qui donne une intersection elliptique avec la section transversale du tumel. A ce titre, il est apparu intéressant de tester sur un cas ( $\varphi=30$ degrés et $C / D$ = 1 en l'occurrencel, un tunnel présentant la même section elliptique que celle correspondant au mécanisme de rupture obtenu par aralyse Jimite (grand axe D = $6.5 \mathrm{~m}$ et petit axe $\mathrm{d}=5 \mathrm{~m}$ ). Dans ce cas, la différence entre la pression limite évaluée par l'analyse limite et celle fournie par un calcul en déplacement n'est plus que de $7 \%$ pout le cas d"un matériau standard (alors qu'elle était de $28 \%$ pour le cas d'un tunnel avec une section circulaire ce même diametrel. Ceci montre qu'une partie de l'écart avec la bontse supérieure don née par le mécanisme à cinq cônes de Soubra (2000) tient au choix du mécanisme lui-nême. Un mécanisme épousant la section circulaire du tunnel (de conception bier plus complexel, permettrait certainement d'améliorer ce résultat.
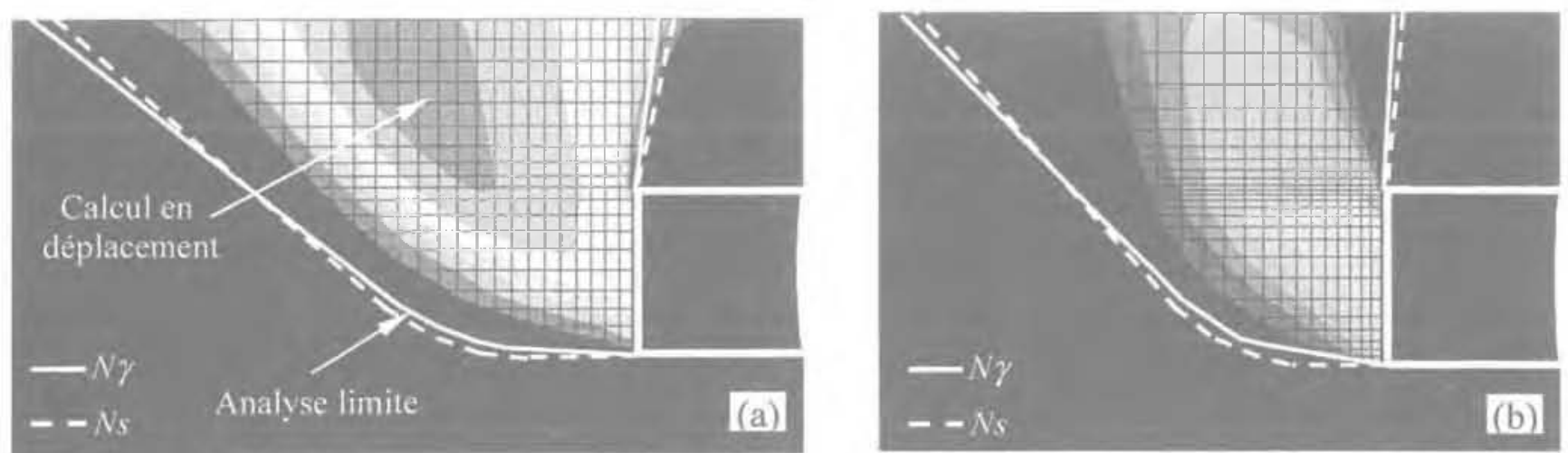

FG 11 Mécanismes en butêe $\varphi=30$ degrés et $C / D=1:(a) \psi=(p:(b) ~ \psi=0$.

Blow-out fallure mechanism $\varphi=30$ degrees and $C / D=$ : : (a) $\psi=\varphi$ : (b) $\psi=0$.

\section{Conclusion et perspectives}

Pour étudier la stabilité tridimensionnelle du front de taile des tunnels à faible couverture et afin d'avoir une estination réaliste de la pression critigue de soutènement du front. des caiculs en déplacement utilisant le code en différences finies explicites FLAC ont été entrepris. Une méthode d'exploitation des résultats et d'analyse des mécanismes de rupture a également été présentée.

Dans le cas des matériaux standard $\{\varphi=\psi \|$, te calcul en déplacement fournit des résultats (jression limite et mécanisme de ruptureł qui restent bien encadrếs par les bornes supélieures des approches tridimensionnelles par analyse limite existantes dans la lirtérature. La comparaison avec l'ensemble de ces approches lqui se distinguent essentiellement par la forme générale des mécanismes de rupture considérés) prouve que ces dermières fournissent, dans le cas de la poussée. une évaluation satisfaisante de la borne supérieure lau maximum $10 \%$ de différencè. En revanche, dans le cas de la butée. elles peuvent conduire à une très forte surestimation de la pression limite, jusqu à $40 \%$ et donc à trop coptimisme. Dans ce detnier cas, le mécanisme de rupture fournit par l'analyse limite encadre largement le champ de vitesse observé.

Le caractère associế ou non de la loi d'écoulement du matériau a un impact très impontant en particuliè sur la butée. Lensemble des calculs en déplacement a montré que si la différence dans le cas d'une rupture par poussée n'est que de $7 \%$ au maximum, en revanche elle peut aller jusqu'a $40 \%$ dans Je cas d"une rupture par butée.

La prise en compte du caractère non associé du comportement du sol n'est théoriquement pas possible par" l'analyse limite. L'approche par analyse limite non associée proposée par Drescher ot Detoumay (1993) a été testée : si elle conduit effectivement à une bonne tpproximation de la borne supérieure dans le cas de la butếe pour $w=0$, il n'en est pas de mëme en poussée oú la nature du niécanisme de rupture est différente.

Le calcul en déplacement a permis de montrer les limites des mécanismes actuellement pris en conpte dans l'approche par l'analyse limite:

- dars le cas extrême du matériau non standard $(\psi r=0)$, le calcul en déplacement foumit un mécanisme de rupture dẻbouchant et cylindrique dans sa partie supérieure 
- la section transversale de la zone en rupture au niveau du front (circulaire pour le calcui en déplacement) n'est pas correctement représentée par l'analyse limite isection elliptique de demi grand axe vertical et égal au diamètre du tunnel).

Le calcul en déplacements peut ainsi être utilisé comme source d'inspiration pour le développement de nouveaux mécanismes en analyse limite.

Enfin, l'ensemble des approches par analyse limite proposées jusqu’è présent, considère un tunnel dont le diamètre est faible au regard de la variation de la pression applicuée au front (par" la bowe ou Ja terre dans les techniques du turnelier à front pressurisé). La pression $\sigma_{7}$ est donc supposée constante. Il serait intéressant de faire évoluer ces approches vers l'anaJyse de tunneis de grands diamètres pour lesquels la rupture pourrait être plus localisée et ne pas intéresser l'ensemble du front. Le calcul en déplacements pourrait alors également servir de base de validation à ces nouvel!es approches.

\section{MBM-}

Les auteurs nemercient R. Kastner. Professeur au Laboratoine de génie

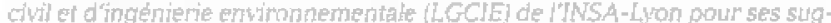

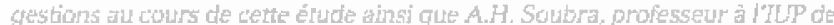

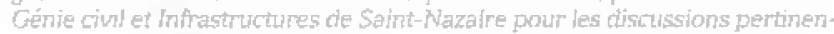

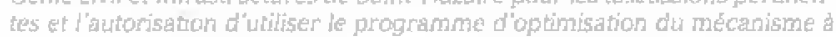
cones. ce qui a permis de réaliser ce travail avec la précision qu îj wertait.

\section{Bibliographie}

Atkinson J.H. Potts D.M.-- Stability of a shallow circular turnel in coltestonless soil. Géotechrique 27, r.2 2, 1977, p. 203. 215.

Chambon P. Corte J.-E - Stabilité du front de taille d'un turnel dans un milieu frot tant, approche cinematígue en calcul à la rupture. Fevue francaise de géotechnicue n ${ }^{\circ} 51,1990$, p. 5 \%-59.

Davis E.H. Gunn M.J., Malr R.J. Senevilatne H.N. - The stability of shallow turnels and underground openings in cohesive material. Géotechaique 30 $n^{0} 4,1980$, p. $397-416$

Demagh R., Benmebarek $S_{\text {, }}$ Kastner R. Analyse $3 D$ des mouvements qénérés par fe front d'un tumbelier à pression de boue. Conférence internationaje GeocityNet05. 2005, Litle, France.

Demagl R., Benmebarek S., Kastner R. Analyse $3 \mathrm{D}$ cles mécanismes de rupture du front de taille dans les sols meubles. Symposium infernationa EL EVLLS. 2006, Marne-]a-Vallée LCPC/ENPC. France.

Drescher A., Detoumay E. - Llmit Joad in translationa! failure mechanisms for associative and non-associative materials. Géotechnigue 43, n 3,1993, p. $443-456$.

Droniuc N., Magnan J.-P. Mestat P. Hum. bert P. - Introduction de la méthode cimématique rếularisée dans CESAFLCPC imodule LIML). Bulletin des Laboratoires des ponts el chaussees $n^{\circ} 256$. 257,2005 . p. 135-148.

Droniuc N., Magnan J.-P., Humbert P., Mestal P. - La nuéthode cinématique régularisẻe appliquée à l'étude de la stabilité des pentes. Bulletir des laboratoires des ponts el chausies n' 256-257, 2005, p. $149-161$

FLAC ${ }^{2 n}$, Fast Lagranglan Analysis of Continua in Three Dimensions. Itasca Consulting Group Inc., 2000, Mineapolis.

Leca E., Panet M. - Application du calcul a la rupture à la stabilité du front de tailie d"un tunnel. Revure francaise de géotech. nicue r ${ }^{\circ} 43,1988,0.5-19$

Leca E., Domieux L. - Upper and lower bound solutions for the face stability of shatlow circular tumels in frictiona. material. Géotennique 40, $1^{\circ} 4,1990$ p. 581-606.
Leca E., Domieux L. - Contribution l'étude de la stabilité du fount de taille d"un tumel en milieu coherent. Rov'w frapcaise de Géotechnique n ${ }^{\circ}$ 61, 1992, p. 5-16.

Lumin A. V. Sloan S. W. - Upper bound limit analysis using linear finte alements and nonminear programming. Int. J. Numer. Anal. Moth. Goomect. 26. 2002, p. 181-216.

Muelhaus H. B. - Lower bound solutions for circular turinels in two and threa dimensions. Rock Mechanics and Rock Engineering 18, 1985, p. 35-52.

Pastor J. Thai T.H ef Francescato P - New bounds for the height linit of a vertical slope. Int. J. Numer. Anal. Meth. Geomech. 23, 2000, p. 165-182.

Soubra A.H. - Three-dimensional face stability analysis of shallow circular tunnel. International Conference on Geotechnical' and Geological Engineering. Melboume, Australia, 2000, p. 1-6.

Wong H., Subrin D. - Stabilité frontalo d'un tungel: mécanisme $3 \mathrm{D}$ en forme de corne et influence de la profondeur. Revue elmopenne de génie civil. vol. 10 $n^{\circ} 4,2006$, p. 429456 . 